\title{
La pedagogía como ciencia: su objeto de estudio, categorías, leyes y principios
}

Omar Abreu-Valdivia1, Ramón Pla-López², Miguel Naranjo-Toro ${ }^{1}$ y Soraya Rhea-González ${ }^{1}$

(1) Universidad Técnica del Norte, Campus. Avenida 17 de julio, 5-21, General José María Córdova, Código postal 100105, Ibarra, Ecuador (correo-e: oabreu@utn.edu.ec, menaranjo@utn.eu.ec, srheag@utn.edu.ec)

(2) Universidad “Máximo Gómez", Ciego de Ávila, Cuba (correo-e: rplalopez@sma.unica.cu)

Recibido Nov. 24, 2020; Aceptado Ene. 22, 2021; Versión final Feb. 10, 2021, Publicado Jun. 2021

\begin{abstract}
Resumen
El principal objetivo del presente estudio fue examinar el sistema teórico de la pedagogía como ciencia desde una perspectiva integradora. Esto requirió de la metodología de la investigación-acción participativa y del estudio documental para la búsqueda de artículos publicados en bases de datos científicas (Web of Science, Scopus, Scielo y Latindex). Se empleó el gestor bibliográfico Mendeley y el criterio de expertos acerca de la utilidad y el carácter objetivo del sistema teórico de la pedagogía. Se examinaron 83 publicaciones, y luego de un proceso de selección basado en sus aportes, se usaron 30. Los resultados evidencian la sistematización de la teoría acerca de la pedagogía como ciencia, la formulación de un sistema teórico que incluye la definición de su objeto de estudio, de sus categorías esenciales, de una ley y de sus principios. Se concluye que la pedagogía es una ciencia que posee un sistema teórico propio integrado, armónico y en desarrollo.
\end{abstract}

Palabras clave: pedagogía; objeto de estudio; categoría; leyes; principios

\section{Pedagogy as a science: object of study, categories, laws, and principles}

\begin{abstract}
The main objective of this study was to examine the theoretical system of pedagogy as a science from an integrative perspective. A participatory research action methodology was applied. Eighty three research articles were retrieved from scientific databases (Web of Sciense, Scopus, Scielo, and Latindex) and the Mendeley bibliographic manager was used. A total of 30 publications were selected based on their contributions. Expert criteria about the usefulness and objective character of the proposed theoretical system of pedagogy were introduced and assessed. The results showed the systematization of pedagogy theory as a science. There was also evidence for the formulation of a theoretical system that includes the definition of pedagogy's object of study, essential categories, laws, and principles. It is concluded that pedagogy is a science that possesses its own integral, harmonic, and developing theoretical system.
\end{abstract}




\section{INTRODUCCIÓN}

El sistema teórico de la pedagogía constituye el núcleo de este trabajo, cuya vigencia gira en torno a imprecisiones, diversidad de criterios, confusiones y divagaciones que no permiten discernir entre pedagogía, didáctica (Abreu et al., 2018), educación escolarizada y no escolarizada y los procesos que se desarrollan en la primera (Abreu et al., 2020). Olesko (2006) afirma que los estudios al respecto han florecido en los últimos años, pero Lederman (2010) piensa que este tipo de debate es insuficiente pues la pedagogía ha tenido que recorrer un camino de altibajos e incertidumbres como campo científico. Para Mendoza (2004) la pedagogía es una disciplina científica dispersa, un proyecto en construcción, lo que da validez a reflexiones como la presente, acerca de su objeto, su estructura, su capacidad explicativa, su sistematicidad, verificabilidad y sus lagunas e indefiniciones.

Su (2014) manifiesta que los académicos de la pedagogía han discutido su cientificidad durante años y que se han formado dos escuelas; una que sostiene que es ciencia y otra que argumenta que no lo es, cada una con sus razones. Considera que la investigación de la pedagogía no se puede hacer sin los estudios sociales, por lo tanto, no es una ciencia o en el mejor de los casos es una ciencia inmadura. La poisción de Moreno (2009) es más radical, afirma que no es una ciencia, sino un lenguaje sin significado, un discurso vacío, carente de contenido y una jerga resistente a usar el método científico, con multiplicación de palabras, da nuevos nombres a cosas que ya tenían uno y multiplica "tonterías" que chocan contra el buen sentido de cualquier profesor.

En opinión de Pérez et al., (2015) esta polémica exige una ardua labor de reflexión, verificación y contraste de los implicados en el estudio y la práctica, para elaborar una terminología común. Para ellos construir los fundamentos de una ciencia o disciplina científica, no es una tarea fácil y agregan que los agentes del estudio social no han adquirido la capacidad y de pensar con rigor, lo que es fundamental para una profesión en proceso de maduración. Korzhuev, et al., (2018) afirman que se ha acumulado una capa sólida de conocimiento pedagógico, pero no se interpreta adecuadamente.

Lo cierto es que la pedagogía es una ciencia que posee un objeto de estudio, un sistema de categorías, leyes y principios que se perfecciona en medio de una variedad de terminología, dispersa, contradictoria e imprecisa. Acerca de su objeto de estudio existen criterios que evidencian la falta de unidad y de integración teórica en torno al asunto. Una situación parecida ocurre con el resto de los componentes del tema que se desarrolla. Las categorías y los principios son menos tratados. Las leyes son abordadas con más insistencia, aunque con menos profundidad, y aparecen más ligadas a la didáctica que a la pedagogía, una especie de sugerencia equivocada de que ambas son lo mismo y no lo son (Ramos et al., 2017), cada una tiene su propia esencia, objeto de estudio, función y sistema teórico en pleno desarrollo (Abreu et al., 2017)

La educación es un fenómeno de alta complejidad Nind y Lewthwaite (2018) inherente a la sociedad desde que esta existe. Cuando el ser humano comenzó a pensar desde la ciencia, no existían la pedagogía ni la didactica; por tanto, los primeros estudios del fenómeno educativo se hicieron desde la filosofía. Después se integraron la sociología, la antropología, la economía, la psicología y la biología, entre otras, y dieron origen a disciplinas como la filosofía de la educación, la sociología de la educación, la psicología educativa y la antropología de la educación. El caracter social y complejo de la educación demanda que se estudie por diferentes disciplinas científicas. Sin embaro, cuando es organizada, estructurada con una intencionalidad bien definida, con contenidos, métodos y técnicas prifesionales seleccionados en función de esa intencionalidad, aparecen la pedagogía y la didáctica. La figura 1 expresa la dinámica de esta relación.

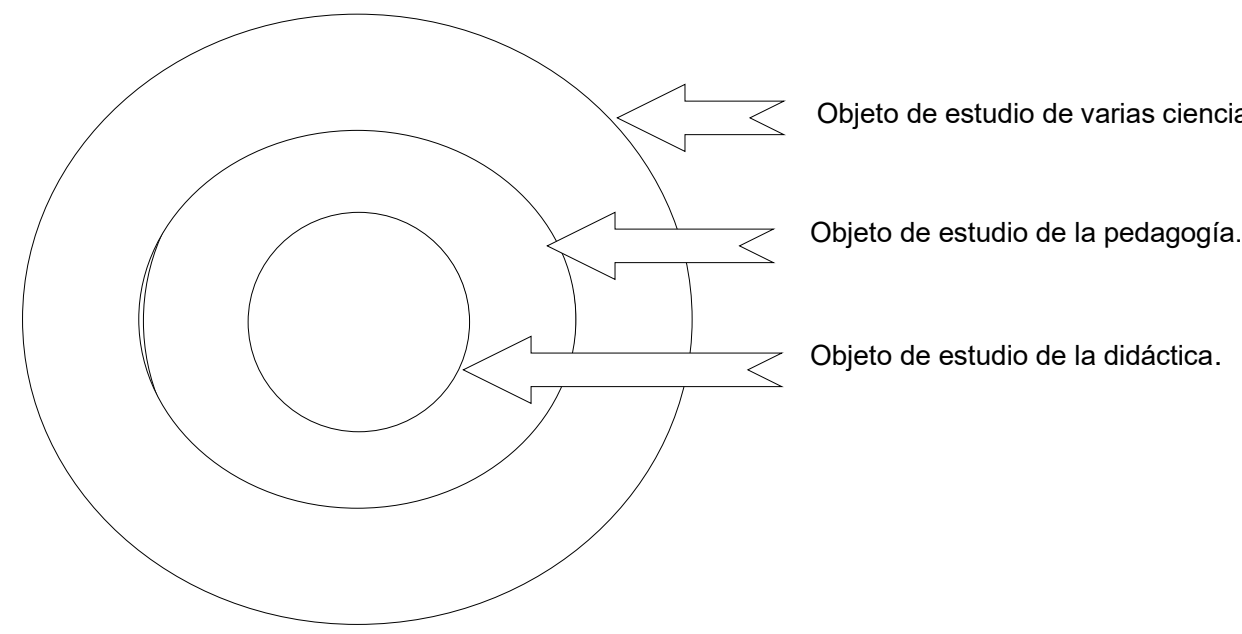

Fig. 1. El proceso educativo, el proceso de enseñanza-aprendizaje y las ciencias que los estudian 
El propósito de este artículo es someter al criterio de la comunidad científica vinculada con el tema que desarrolla, un sistema teórico de la pedagogía como ciencia, contrastado con la teoría precedente y que consiste en la definición de un objeto de estudio exclusivo, la selección y fundamentación de seis categorías, algunas propias y otras compartidas, la formulación de una ley general y de seis principios, desde una posición dialéctica integradora.

\section{METODOLOGÍA}

Se aplicó el estudio documental para evaluar e interpretar investigaciones relevantes acerca de la pedagogía publicadas en bases de datos científicas como: Web of Sciense, Scopus, Scielo y Latindex. Se utilizaron algunos libros evaluados por pares que no se encuentran en estas bases de datos cuyos autores tienen reconocido prestigio dentro del tema. El gestor bibliográfico Mendeley se puso en práctica para almacenar, organizar y analizar las fuentes consultadas. Se emplearon términos o palabras clave como: pedagogía, didáctica, ciencia, objeto de estudio, categorías pedagógicas, leyes pedagógicas, principios pedagógicos, proceso educativo (PE) y proceso de enseñanza-aprendizaje (PEA) para facilitar la búsqueda. Se estudiaron 83 publicaciones y luego de un proceso de selección basado en sus aportes, finalmente se usaron 30 como referencias, el $86.6 \%$ de las cuales pertenece a Web of Sciense y Scopus y el $13 \%$ a Scielo y Latindex. Se incorporaron 2 libros que no están en estas bases, pero son publicaciones especializadas evaluadas por pares cuyos aportes son útiles para el debate. El $70 \%$ de las fuentes seleccionadas corresponde a los últimos 10 años y el $60 \%$ a los últimos 5 .

La metodología de la investigación-acción participativa facilitó la construcción del sistema teórico de la pedagogía como ciencia que se propone y que fue enriquecido sistemáticamente por los investigadores, siguiendo una espiral de ciclos de revisión bibliográfica, planificación, selección de contenidos, análisis, acción, observación, reflexión, debate, depuración y construcción colaborativa. Esta metodología sirvió para perfeccionar otros sistemas hasta entonces vigentes en las universidades donde se desarrolló la investigación. Los autores desempeñaron los roles de investigador y participante e integraron conocimientos teóricos y prácticos en su contexto educativo, asumiendo posiciones críticas y autocríticas, socializando y enriqueciendo los resultados.

Se introdujo el criterio de expertos aplicado a 15 profesionales de diferentes nacionalidades con títulos de $\mathrm{PhD}$ en ciencias de la educación, con más de 10 años de experiencia en el ejercicio de la docencia, para recopilar el criterio de estos profesionales acerca de la utilidad y el carácter objetivo del sistema teórico de la pedagogía propuesto. Las alternativas de evaluación oscilaban entre el 1 y el 5 , en una escala ascendente, para los siguientes indicadores,1) vigencia del tema, 2) referentes utilizados, 3) lógica de la propuesta, 4) argumentos de la pedagogía como ciencia, 5) definición del objeto de estudio, 6) definición de las categorías, 7) formulación de la ley, 8) formulación de los principios, 9) visión integradora del PE, 10) coherencias del sistema propuesto, 11) novedad del sistema teórico propuesto y 12) utilidad académica, los cuales fueron evaluados como muy adecuado (MA) bastante adecuado (BA) adecuado (A) poco adecuado (PA) y no adecuado (NA). La figura 2 expresa el resultado de la evaluación de los expertos sobre la base de los indicadores definidos.

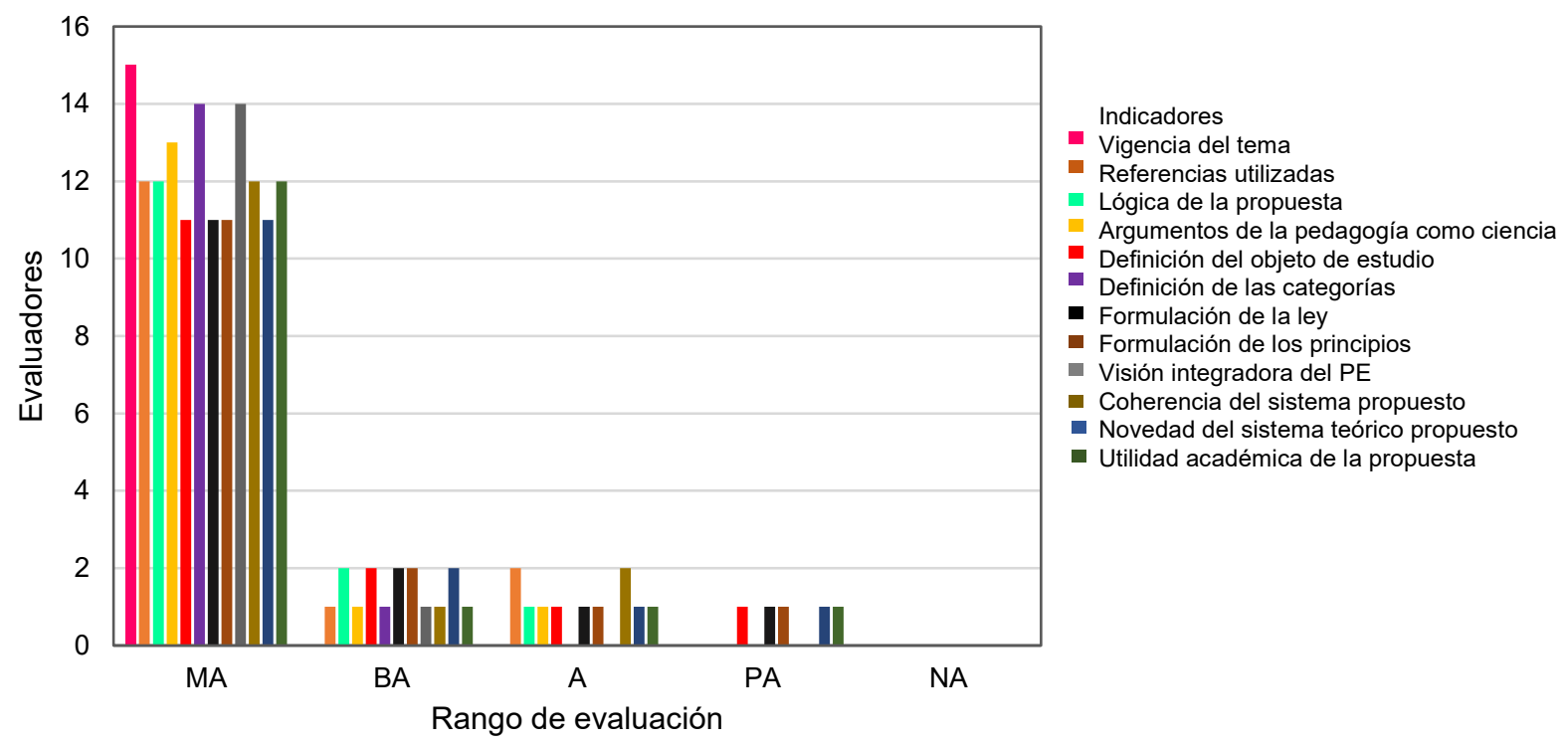

Fig. 2. Resultado de la evaluación de los expertos. 


\section{RESULTADOS}

El análisis de la información de la figura 2 confirma la opinión favorable de los expertos acerca del sistema teórico propuesto. El indicador vigencia del tema fue evaluado positivamente por el $100 \%$ de estos profesionales. La definición de las categorías y la visión integradora del PE por el $93.3 \%$. Los argumentos de la pedagogía como ciencia recibieron el $86.6 \%$ de aceptación. Otros como referentes utilizados, lógica de la propuesta, coherencias del sistema y utilidad académica fueron beneficiados con el $73.3 \%$ de consentimiento. La definición del objeto de estudio, la formulación de la ley y de los principios y la novedad del sistema teórico tuvieron una acogida de un $73.3 \%$.

La formación del ser humano ocurre en el marco de la educación, escolarizada y no escolarizada. La primera se manifiesta mediante el (PE) y este a su vez incluye al PEA. El PEA es el objeto de estudio de la didáctica (Abreu et al., 2018) y el PE lo es la pedagogía (Ramos et al., 2017). Sin embargo, el PE referente debe reunir determinadas características para ser definido como el objeto de estudio de esta ciencia. La figura 3 revela la dinámica de esta relación.

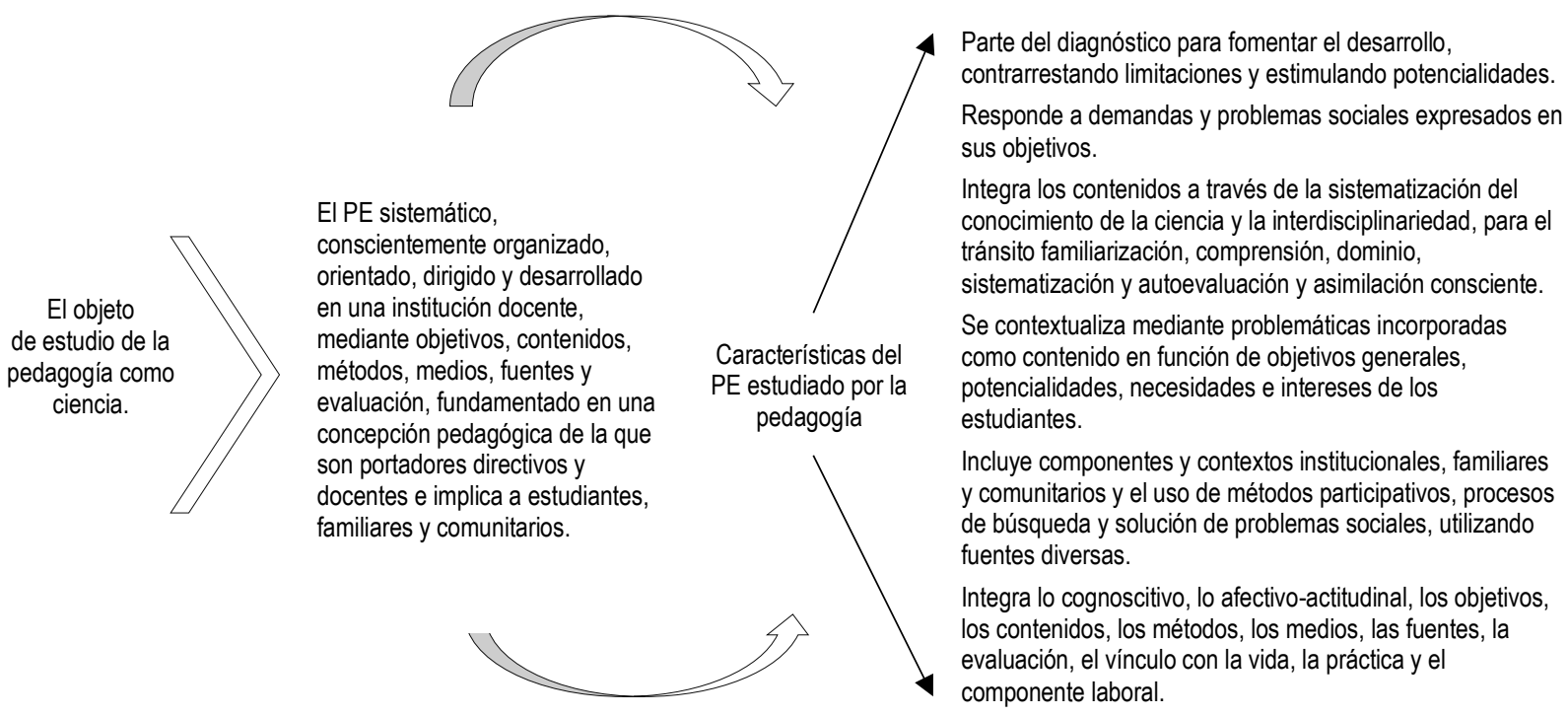

Fig. 3. Representación del PE como objeto de estudio de la pedagogía.

\section{Las categorías y conceptos de la pedagogía}

Las categorías son conceptos de máximo nivel de generalidad de una ciencia. Reflejan los fenómenos y leyes más holísticas de la rama del saber dicha ciencia.

\section{Educación e instrucción}

Educación es la categoría de mayor esencialidad y generalidad de la pedagogía. Es la preparación del ser humano para insertarse en la sociedad y desarrollarla. Requiere el aprendizaje de conocimientos, habilidades, valores, métodos y modos de actuación a través de actividades organizadas como un proceso sistemático, intencionalmente concebido, relacionado con fines de la sociedad, con contenidos especialmente seleccionados, para generar una dinámica comunicativa estructurada, temporal y particular, sobre la base de las características individuales y colectivas de los sujetos, lo que requiere una orientación pedagógica por parte de educadores. La instrucción es una categoría que se utiliza dentro del sistema teórico de la pedagogía, para designar una dimensión de la educación referida a la asimilación de conocimientos y habilidades y está integrada a la categoría educación. Entre ambas existe una unidad dialéctica.

Ambas están relacionadas de manera históricamente cambiante, lo mismo que los fenómenos que reflejan, lo cual se corresponde con el contenido de la educación. La formación del sentimiento (educación) está inmersa en la del pensamiento (instrucción) y a la inversa. La educación es más general que la instrucción; no se limita a la formación moral y conductual, sino que integra el resultado del PE y se alcanza mediante la instrucción, dirigida de manera consciente y viceversa. La instrucción es una dimensión de la educación que no puede darse aisladamente del resto de las influencias. Durante el siglo XVIII y primera mitad del siglo XIX no se distinguían. Se hablaba de instrucción del corazón (educación) y de instrucción del intelecto (instrucción). También se planteó que la diferencia entre ambas estaba en la coerción. La educación se veía como influencia coercitiva, impuesta por una persona sobre otra y la instrucción como una relación libre para adquirir información. 
La formación del sentimiento (educación) está inmersa en la del pensamiento (instrucción) y viceversa. La educación es más general que la instrucción y, por tanto, es un resultado a más largo plazo. De tal manera, la educación como resultado se alcanza mediante la instrucción, dirigida de forma consciente y viceversa. La instrucción es una dimensión de la educación y no puede darse aisladamente del resto de las influencias. Esta forma de asumir la relación instrucción educación fundamenta la posición de considerar a la categoría educación como la más esencial, es decir como principal y más general de la pedagogía, que no se limita a la formación moral y conductual, sino que integra el resultado del PE.

\section{Formación y desarrollo}

La formación y el desarrollo son asumidas por la pedagogía para explicar el PE de la personalidad de los alumnos que se desarrolla en la institución. La categoría desarrollo está asociada a la formación. No obstante, la formación y el desarrollo tienen sus propias características. La primera responde a las regularidades internas del proceso de que se trate, sin que implique desconocer la influencia socio-educativa y la segunda está más ligada a las que se encuentran en la base del PE. Ambas implican la consideración del hombre como un ser bio-psico-social.

Formación y desarrollo, introducidas en la pedagogía desde otras ciencias, no son de su sistema categorial de máxima generalidad, sino conceptos que permiten describir resultados de aprendizajes, habilidades, valores, capacidades, competencias y facetas en las formaciones psicológicas de la personalidad y son necesarias para comprender el progreso de la personalidad que se educa, identificar resultados parciales del aprendizaje y para referirse al fin de la educación como contribución a la formación y al desarrollo de la personalidad. La pedagogía no controla la formación y el desarrollo de la personalidad desde su objeto, pero contribuye con ellos.

\section{Comunicación y actividad}

La comunicación y la actividad forman parte de la definición de educación y del PE. Se originan en la filosofía y la psicología los desarrolla. La educación, la enseñanza y el aprendizaje ocurren en la actividad y a través de la comunicación. La comunicación educativa debe caracterizar al PE. Se distingue por la participación activa de los alumnos, la significación de la información y el estilo democrático. En ella intervienen educadores, familiares, alumnos, directivos, representantes de las organizaciones de la institución educativa y otros integrantes de la comunidad, los cuales son transformados en un proceso de crecimiento personológico, matizado por cierto carácter planificado y orientado en dependencia de los objetivos planteados.

La actividad educativa es particular del PE, desarrollada por los alumnos y orientada por educadores. Tiene lugar en los diferentes contextos de actuación, en el PEA y en otros de la institución o fuera de ella, siempre bajo conducción de esta. Se diseña, ejecuta y controla a partir del diagnóstico, teniendo en cuenta los objetivos y contenidos para un nivel o grado y adquiere un proceder dinámico que responde a leyes y principios de la pedagogía.

\section{Ley pedagógica del PE}

La ley se asocia a reglas o normas que expresan constancia, sistematicidad e invariabilidad de ciertas cosas. Manifiesta la dialéctica entre los componentes de un fenómeno. La pedagógica expresa la conexión interna, esencial y estable de los componentes del PE, en el que se producen las interacciones del alumno con la realidad, con las demás personas, organizadas en etapas y fases interdependientes, conforme a las necesidades sociales y contextuales. Considerando el objeto de estudio de la pedagogía, las categorías y conceptos principales, las relaciones principales entre los componentes personales y personalizados asumidos, se elaboró una ley pedagógica integradora que expresa lo siguiente: el PE es patrimonio de instituciones educativas, está condicionado histórica y socialmente, es planificado, organizado, dirigido y desarrollado pedagógicamente, exige una relación dialéctica entre sus agentes personalizados (educador, educando) mediante objetivos, contenidos, métodos, fuentes y evaluación y una dinámica desarrolladora, enseñanzaaprendizaje, en diferentes escenarios educativos, en función de la formación integral y contextualizada de la personalidad durante la vida.

La formulación de esta ley distingue cualitativamente la educación desde el PE y revela la existencia de un proceso conscientemente organizado y conducido, condicionado por las relaciones sociales predominantes en una época determinada. La dinámica interna que se expresa en ella se entiende como las interacciones entre los componentes personales de este proceso, en las que el modo de actuación del educador es esencial para lograr los objetivos. La función del educador en la selección de contenidos, métodos, formas organizativas, medios, fuentes y evaluación, no desconoce el rol activo de los estudiantes y de otros componentes con los cuales interactúa. 


\section{Principios pedagógicos del PE}

Para que la ley se corresponda con resultados deseados, se elaboran principios que expresan lo que se quiere lograr en un sistema o grupo social. El principio es una guía, regla, norma, reflejo de la necesidad o de la ley para la acción o el pensamiento. La comprensión de una ley inherente al PE y de la necesaria y consecuente diversidad de sistemas de principios es un avance para la pedagogía. Considerando la sistematización de la información consultada, las posiciones epistemológicas, la ley pedagógica y la práctica educativa, se propone un sistema seis de principios pedagógicos del PE, integrados a las condiciones socio-históricas de la educación.

\section{El carácter científico y la intencionalidad de la educación}

Educar no es trasmitir adelantos de la ciencia y la técnica a los alumnos, sino desarrollar en ellos una concepción científica del mundo, acerca de la sociedad, la naturaleza, los seres humanos, el pensamiento y sobre sí mismos, que se convierta en convicciones y núcleo de su formación. Uno de los objetivos centrales de la educación en la actualidad es la asimilación por el alumno de los conocimientos científicos de la época contemporánea y la formación de una concepción y actitud científica hacia los fenómenos de la realidad, de un pensamiento científico y de una actitud responsable ante el conocimiento y su empleo en función del bien social, lo cual se logra mediante la unidad entre el carácter científico y la intencionalidad de la educación.

\section{Integración entre el carácter social y el individual}

En el PE se produce cierta contradicción entre su influencia individual y su ejecución en forma grupal. Mientras se desarrolla la docencia se ejecutan actividades grupales, hacerlo individualmente sería imposible, pero se aspira a que la influencia sea estudiante individualmente en cada. El colectivismo es una de las cualidades que debe tener un alumno, para ello es necesario que se logre el fortalecimiento de la solidaridad, el respeto mutuo, la disposición a la ayuda, la franqueza, el trabajo colaborativo, el diálogo, el debate, la cooperación desinteresada, la actitud crítica y la autocrítica. Un colectivo escolar no es un agregado de personas, sino una entidad viva con la cual el educador interactúa para aproximarse a cada integrante. La educación exige una atención grupal (el individuo como parte de distintas agrupaciones) y otra individual (como personalidad) pues ambas se complementan. La educación debe tener un enfoque personológico, centrar el PE en la personalidad de los educandos y los educadores y en su carácter activo, al considerarlos componentes personales y sujetos de la actividad pedagógica, con características concretas, irrepetibles, en un nivel sintético-unitario.

EI PE está dirigido a personas en diferentes etapas de su ontogenia, a personalidades en desarrollo, cuyas características son punto de partida para la formación. En el PE influyen factores exógenos y endógenos, la familia, otras personas y el contexto, por lo que debe existir una relación complementaria entre todos para que el influjo se corresponda con las funciones de cada uno. La unidad es sinónimo de integración, de unión, conformidad y unanimidad racional. Cada educador debe ser consciente de la función que le corresponde en la formación del estudiante, para que no existan diferentes niveles de exigencia y de dirección del aprendizaje en un mismo grupo escolar, excepto las que justifican la atención a las diferencias individuales.

\section{Unidad entre lo afectivo y lo cognitivo}

El ser humano no permanece impasible ante el mundo que le rodea, adopta una posición concreta ante él. Los elementos que se integran en la personalidad tienen una esencia cognitiva y afectiva, por lo que resulta difícil delimitar un fenómeno psicológico puramente afectivo o cognitivo en ella. Los conocimientos y habilidades de sentido personal para el estudiante, provocan una regulación efectiva de su conducta y viceversa, por tanto, los motivos proclives a la escuela y al aprendizaje facilitan la asimilación de los contenidos científicos, el desarrollo de habilidades y la formación de valores. Sin embargo, el conocimiento no implica automáticamente la manifestación conductual, sino cuando es relevante en su reflejo afectivo volitivo, por ello las operaciones cognitivas deben incluir un contenido emocional favorable para los logros educacionales y los estándares básicos, de ahí que el docente deba imprimirle una alta carga afectiva y motivacional al PE.

\section{El carácter consciente, activo e independiente de los sujetos}

Consiste en asegurar la correlación entre la dirección pedagógica y el trabajo consciente y creador de los alumnos. Se trata de emplear la ley para lograr que estos dominen conscientemente los conocimientos científicos y los métodos para su aplicación, demostrando iniciativa, interés, creatividad y sentido crítico valorativo, para una la actividad consciente y creadora, el desarrollo del pensamiento lógico y la formación de una concepción del mundo. Las exigencias externas en la educación están sujetas a la individualidad, a las condiciones internas del sujeto, a sus conocimientos, habilidades, concepciones, criterios éticos, motivaciones 
e intereses y costumbres. Solo un PE que tenga presente las ideas, la voluntad y el estado de ánimo de los alumnos, despierte sus dudas, reflexiones, inquietudes, inconformidades, satisfacciones y esfuerzos, contrarreste sus limitaciones y modifique sus potencialidades, será capaz de ejercer las influencias necesarias.

Este principio no niega el rol orientador y guía del educador. Expresa un nuevo tipo de relación, en el cual el trabajo de los estudiantes es activo y consciente e inicia con su implicación en las tareas, necesidades, futura profesión y el surgimiento del interés por realizarlo acertadamente. Una vía para consolidar la necesidad interior de conocimientos, habilidades y valoraciones es la creación de condiciones que refuercen la contradicción entre ideas y nociones de los estudiantes sobre uno u otro objeto, proceso o fenómeno; entre su experiencia vital y un nuevo conocimiento científico.

\section{La comunicación educativa y la actividad educativa}

Se establece como una regularidad la unidad entre las categorías actividad y comunicación debido a la estrecha relación entre ambas en la formación del ser humano, pues son dos procesos psicológicos muy vinculados, en el desarrollo de la personalidad del estudiante. La realización de actividades conjuntas condiciona la necesidad de la comunicación entre las personas, en la medida que sea mayor y más eficiente esa comunicación, con más efectividad se logran los objetivos de la actividad. El desarrollo de la personalidad del estudiante exige una adecuada y armónica unidad entre las actividades que realiza (relación sujeto objeto) y la comunicación que se establece con los demás. Una de las condiciones para el éxito de la labor educativa radica en la calidad de las actividades que emprenden los estudiantes junto al educador para la apropiación del contenido y la comunicación asertiva que establezcan ambos y los estudiantes entre sí.

\section{Unidad de las influencias de los contextos de interacciones}

El aprendizaje es resultado de la asimilación histórico concreta de la cultura, por tanto, mientras más variada y profunda sea la correlación del alumno con el contexto, más completa y eficiente será su formación. La educación se organiza mediante el PE y no debe limitarse al PEA porque a pesar de ser su núcleo no puede garantizar por si solo toda gama de aprendizajes que la educación exige. El primero debe estructurarse considerando otros escenarios que favorezcan aprendizajes que solo se obtienen parcialmente en el segundo. EI PEA es el centro del PE y del sistema de intercambios que en él se producen, debe estar en unidad y coherencia con las interacciones de contextos y actividades, productivas, político sociales, culturales, deportivas, humanitarias, entre otros, entendiendo que todo tiene lugar en seno de la institución, la familia y la comunidad.

Un educador debe desarrollar habilidades profesionales mediante la pedagogía para integrar acciones educativas en diferentes contextos. La más integradora para este fin es el diseño y orientación de proyectos educativos fundamentados por la teoría pedagógica, concebidos, ejecutados y evaluados en una institución o grupo escolar, bajo la conducción científica de docentes y directivos, para elevar la calidad de la formación integral de los estudiantes, quienes protagonizan su propia educación con la participación de la comunidad escolar, a partir de problemas priorizados, determinados mediante un diagnóstico integral, contextualizado y sistemático.

\section{DISCUSIÓN}

El objeto de estudio de la pedagogía. Los estudiosos no concuerdan ni han encontrado un punto de equilibrio acerca de un sistema teórico pedagógico. Para unos el centro de atención de la pedagogía es la educación en sentido general, otros afirman que es el proceso docente educativo, algunos que es el PEA, no faltan quienes dicen que trata aspectos relacionados con el proceso de aprendizaje a solas, tampoco aquellos plantean que consiste en la educabilidad del hombre ni los que aseguran que es el proceso de enseñanzaestudio-aprendizaje. Calzadilla (2004) afirma que la pedagogía estudia el descubrimiento, apropiación cognoscitiva y aplicación adecuada y correcta de las leyes y regularidades de los procesos de aprendizaje, conocimiento, educación y capacitación y el conocimiento de las acciones para que tales procesos resulten eficientes y eficaces. Para Mendoza (2004) el campo de la pedagogía es el saber trascendente que, por integrador y sintetizador, genera un campo sustentado en una nueva racionalidad, unidad dentro de diversidad, planteada sobre la base de un reconocimiento y respeto de la individualidad humana y de las posibilidades y limitaciones del desarrollo físico, cognitivo, espiritual y moral del ser humano. Para Taber (2017) la pedagogía estudia la educación. Garrido et al., (2020) tienen una opinión similar y añaden que es un fenómeno que se investiga mientras se manifiesta en diferentes contextos por quienes lo examinan y por los que lo poseen. Al decir de Korzhuev, et al., (2018) la pedagogía es una teoría basada en la generalización de la experiencia específica relacionada con la educación. Mynbayeva et al., (2018) mezclan varios aspectos cuando dicen que es la ciencia de la crianza, la enseñanza, el aprendizaje y la educación. 
Pérez, et al., (2014) consideran que centra la atención en el PE y hablan de la percepción de los estudiantes en el mismo. Martí et al., (2018) también defienden este punto de vista. Tirri y Toom (2020) dicen que su objeto de estudio es el proceso de enseñanza-estudio-aprendizaje. Según Fiogla (2018) se ocupa de los vínculos entre el alumno, el profesor y el contenido en el PEA. Gómez (2018) relata que la acción pedagógica es un hecho humano visible, tangible, sistemático, ejercido por el docente en el PEA. Kulabekova (2019) plantea que estudia la transferencia de las tradiciones y costumbres, la interacción de generaciones, es decir, todo lo que fue creado en el desarrollo histórico y la herencia espiritual de la gente. Munck (2007) se refiere al PEA como el objeto de estudio de la pedagogía y la considera ciencia de los profesores. Antoniou (2020) también se integra a esta tendencia y habla de la necesidad de nuevas tecnologías educativas y de pedagogías digitales y Ye (2020) alude a la pedagogía basada en tecnología digital para la interpretación de información en dicho proceso.

Las categorías. Se considera que la educación e instrucción, la formación y el desarrollo y la comunicación y la actividad son esenciales dentro del desarrollo del PE. La literatura consultada no ofrece muchas alternativas para el debate. No obstante, Aparici y Silva (2012) destacan la importancia de la comunicación dentro de este proceso y señalan la necesidad de contrarrestar la que tiene un carácter transmisivo, caracterizada por su sentido unidireccional, por la interactiva.

Las leyes. Para formular las leyes del PE se han empleado estructuras conceptuales diversas y diferentes enfoques sobre el PE, al margen del condicionamiento histórico, social, económico y pedagógico de los sistemas educativos, lo que ha dado al traste con sus posibilidades de éxito. Una secuencia cronológica revela que para Alvarez (1999) son la escuela en la vida, en la que se concreta la relación: problema - objeto objetivo y la educación a través de la instrucción, en la que se concreta la relación: objetivo - contenido método. Para Fuentes (2000) son el vínculo del proceso docente educativo con la sociedad y la dinámica interna de este. Según Herrera (2004) se manifiestan en la dinámica de las relaciones entre problema objeto - objetivo y en las relaciones objetivo-contenido-método. Calzadilla (2004) reconoce solo al arte en su concepción de ciencia humanista. Pla, et, al (2010) proponen la relación entre la enseñanza y el aprendizaje y entre los componentes del proceso de enseñanza-aprendizaje, ambas resultantes de la impartición de las asignaturas y González (2016) señala la relación configuracional entre la sociedad y la educación y el sistema complejo compuesto por varios subsistemas con interacciones configuracionales. A pesar de las discrepancias, existe una tendencia a reconocer el vínculo de la educación con la sociedad y que el PEA posee una estructura y dinámica interna.

Las leyes del PE son objetivas, se manifiestan como expresión de las relaciones esenciales entre las propiedades y componentes del proceso y de las tendencias de su desarrollo, independientemente de la conciencia de sus protagonistas. Rigen la evolución de los procesos, de ahí su valor metodológico para investigarlos, comprenderlos, explicarlos y transformarlos. La educación y las leyes del PE son fenómenos sociales, depende de condiciones socio históricas y expresan la relación causa-efecto. Su conocimiento permite que los efectos pasen de la esfera de lo posible, a la de lo real. La conducción científica del PE presupone que los educadores conozcan sus leyes para limitar o ampliar el radio de actuación de las mismas en conformidad con sus intereses y necesidades. Las leyes pueden ser ignoradas o desconocidas por los educadores, pero no dejarán de manifestarse. Desconocerlas implica actuar a ciegas, pero no impedir su acción; conocerlas, ayuda a conducir científicamente el proceso.

Los principios. Entre las fuentes estudiadas no abundan propuestas de principios pedagogicos para el PE. Atjonen et al., (2011) aseguran que los principios pedagógicos clave se centran en los métodos de enseñanzaaprendizaje, la relación con los alumnos, el entorno de aprendizaje, las acciones educativas generales y las habilidades y actitudes. Herrera (2004) los asocia con el diagnóstico, la motivación hacia el objeto de estudio, la capacitación para la solución de problemas profesionales, la activación para la búsqueda, exploración y reflexión del contenido, la vinculación de este con los procesos, la atención a las diferencias individuales, la interacción del trabajo individual con el colectivo y el desarrollo del autoaprendizaje.

Los principios expresan la aspiración de la sociedad acerca de los resultados del PE, los cuales están dentro de la ley; son consecuencia de esta e indican la dirección de las acciones para el efecto deseado. Son la idea que guía, la regla que orienta la conducta del educador y la actividad del alumno y determinan la efectividad del PE. También son circunstanciales y dependen de la voluntad humana. Constituyen aspiraciones sociales que se modifican en función del conocimiento de la ley. Se consideran temporales, subjetivos y pueden ser o no cumplidos. Su conocimiento, desconocimiento, incumplimiento, cumplimiento parcial o total influye en los resultados de los procesos, en relación con las necesidades y aspiraciones sociales. Los sistemas educativos y las instituciones deciden sus principios y estos serán válidos mientras se correspondan con las necesidades de la sociedad. En tanto un sistema de principios parta de la comprensión de la ley y de las exigencias sociales y logre una mejor comprensión y aplicación por los educadores, desempeñará una mejor su función orientadora para el desarrollo exitosos del PE. 


\section{CONCLUSIONES}

La pedagogía es una ciencia que posee un sistema teórico propio integrado, armónico y en desarrollo que tiene como objeto de estudio al PE de la institución educativa, categorías lideradas por la educación como la de máxima generalidad, una ley que revela la naturaleza de dicho proceso y principios contextualizados que caracterizan las relaciones entre sus agentes personalizados. La compresión y puesta en práctica de este sistema, de manera flexible y creativa, es la base de la educación en función de la formación integral del ser humano.

\section{REFERENCIAS}

Abreu, O., Rhea, S., Arciniegas, G., y Guevara, S., Competencia para el diseño y la ejecución del proceso de enseñanza-aprendizaje en la facultad de ciencias administrativas y económicas, Universidad Técnica del Norte de Ecuador, http://dx.doi.org/10.4067/S0718-50062020000400153, Formación Universitaria, 13(4), 153-164 (2020)

Abreu, O., Rhea, S., Arciniegas, G. y Rosero, M., Objeto de estudio de la didáctica: análisis histórico epistemológico y crítico del concepto, https://dx.doi.org/10.4067/S0718-50062018000600075, Formación Universitaria, 11(6), 75-82 (2018)

Abreu, O., Gallegos, M., Jácome, J., y Martínez, R., La didáctica: epistemología y definición en la facultad de ciencias administrativas y económicas de la Universidad Técnica del Norte del Ecuador. https://dx.doi.org/10.4067/S071850062017000300009, Formación Universitaria, 10(3), 81-92 (2017)

Atjonen, P., Korkeakoski. E. y Mehtäläinen, J., Key pedagogical principles and their major obstacles as perceived by comprehensive school teachers, https://doi.org/10.1080/13540602.2011.554698, Teachers and Teaching, 17(3), 273288 (2011)

Antoniou, J., Quality of experience and learning in information systems, incorporation of learning and ethics in characterization of the quality of experience, https://doi.org/10.1007 / 978-3-030-52559-0_2, 1-8, Springer Nature Switzerland AG, Switzerland (2020)

Aparici, R. y Silva, M., Pedagogía de la interactividad. https://doi.org/10.3916/C38-2012-02-05, Revista Comunicar, (38), 51-58 (2012)

Calzadilla, R., La pedagogía como ciencia humanista: conocimiento de síntesis, complejidad y pluridisciplinariedad, Revista de Pedagogía, 25(72), 123-148 (2004)

Fiogla, I., Science of pedagogy: theory of educational discipline and practice, https://doi.org/10.2478/jtes-2018-0013, Journal of Teacher Education for Sustainability, 20(2), 31-43 (2018)

Fuentes, H., Didáctica de la educación superior, $2^{\mathrm{a}}$ edición, INPAHU, 1-256, Bogotá, Colombia (2000)

Garrido, S., de Andrade, U., y Rolim de Lima, J.L., Pedagogy as a lócus of professional training of educators: epistemological and curricular challenges, https://doi.org/10.5212/PraxEduc.v.15.15528.057, Práxis Educativa, 15, e2015528, 1-20 (2020)

Gómez, J.A., Evaluación del impacto del modelo ccción docente como alternativa pedagógica para el manejo del trastorno disocial escolar desde la perspectiva humanística, http://dx.doi.org/10.4067/S0718-

07642018000600143, Información Tecnológica, 29(6), 143-152 (2018)

González, W., Las leyes de la didáctica y la realidad escolarizada ¿necesidad de cambio?, Revista Latinoamericana de Estudios Educativos, XLVI (3), 85-110 (2016)

Herrera, J.L., La didáctica del proceso docente para el desarrollo de la práctica laboral en las empresas, Actualidades Investigativas en Educación, 4, (2), 1-33 (2004)

Korzhuev, A.V., Sokolova, A.S., y otros tres autores., Pedagogical methodology as a fragment of an emerging modern scientific knowledge, Espacios, 39(49), 28 (2018)

Kulabekova, G., Kozybayev,Y., y otros tres autores, Kazakh national pedagogy in the upbringing of boys, Opcion, 35(902), 806-819 (2019)

Lederman, N.G., A partial list of the empirical theoretical literature on subject-specific pedagogy, https://doi.org/10.1111/j.1949-8594.2001.tb18008.x, School Science and Mathematics, (101) 61-80 (2010)

Nind, M., y Lewthwaite, S., Methods that teach: developing pedagogic research methods, developing pedagogy, https://doi.org/10.1080/1743727X.2018.1427057, International Journal of Research \& Method in Education, 41(4), 398410 (2018)

Martí, A., Martínez, R., y Valcárcel, N., La comunicación entre profesores y estudiantes universitarios durante el desarrollo del proceso docente educativo, Educación Médica Superior, 32(2),1-10 (2018)

Mendoza, C., La pedagogía como ciencia: notas para un debate, Investigación y Postgrado, 19(2), 229-240 (2004)

Moreno, R., ¿Es la pedagogía una ciencia?, Foro de Educación, (11), 67-83 (2009)

Munck, M., Science pedagogy, teacher attitudes, and student success, Journal of Elementary Science Education, 19(2), 13-24 (2007) 
Mynbayeva, A., Sadvakassova, Z., y Akshalova, B., Pedagogy of the twenty-first century: innovative teaching methods, https://doi.org/10.5772/intechopen.72341, In New Pedagogical Challenges in the 21st Century - Contributions of Research in Education, InTech, IntechOpen, 4-20 (2018)

Olesko, K.M., Science pedagogy as a category of historical analysis: past, present, and future, Sci Educ, https://doi.org/10.1007/s11191-005-2014-8, 15(7-8), 863-880 (2006)

Pérez, G., García, J.L., y Fernández-García, A., La cuestión social como objeto de estudio: concepto y fundamento, https://doi.org/10.1080/15348431.2015.1007726, Revista de Latinos y Educación, 14(2), 95-105 (2015)

Pla, R., Ramos, J., y otros quince autores, Una concepción de la pedagogía como ciencia desde un enfoque histórico cultural, 1-79, Pueblo y Educación, La Habana, Cuba (2010)

Ramos, J., Rhea, S., Pla, R., y Abreu, O., La pedagogía como ciencia para el tratamiento de los contenidos generales del proceso educativo y la formación de valores, https://dx.doi.org/10.4067/S0718-50062017000600009, Formación Universitaria, 10(6), 77-86 (2017)

Su, Y., Pedagogy: an immature science on the basis of system science, https://doi.org/10.1016/j.sbspro.2014.01.1387, Procedia - Social and Behavioral Sciences, (122), 525-528 (2014)

Taber, K.S., Knowledge, beliefs and pedagogy: how the nature of science should inform the aims of science education (and not just when teaching evolution), https://doi.org/10.1007/s11422-016-9750-8, Studies of Science Education, 12, 81-91 (2017)

Tirri, K., y Toom, A., The moral role of pedagogy as the science and art of teaching, https://doi.org/10.5772/ intechopen.90502, Core Collection, 1-11 (2020)

Ye, H., Interpretation pedagogy based on digital technology, https://doi.org/10.1007/978-3-030-51431-0_76, Advances in Computing and Intelligent Systems, 1233, 521-527 (2020) 